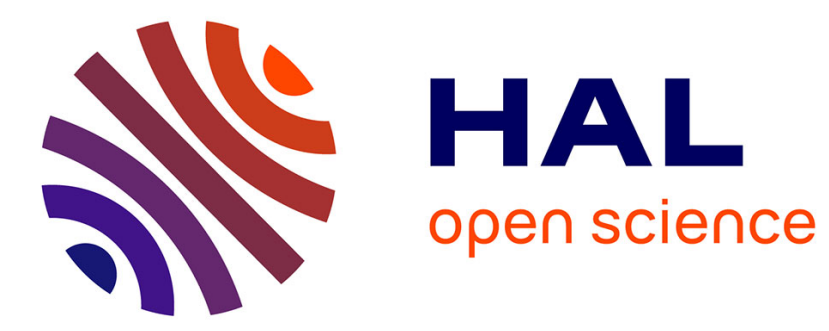

\title{
The ground state tunneling splitting of malonaldehyde: Accurate full dimensional quantum dynamics calculations
}

\author{
Mauricio D. Coutinho-Neto, Alexandra Viel, Uwe Manthe
}

\section{To cite this version:}

Mauricio D. Coutinho-Neto, Alexandra Viel, Uwe Manthe. The ground state tunneling splitting of malonaldehyde: Accurate full dimensional quantum dynamics calculations. Journal of Chemical Physics, 2004, 121 (19), pp.9207-9210. 10.1063/1.1814356 . hal-01118369

\author{
HAL Id: hal-01118369 \\ https://hal.science/hal-01118369
}

Submitted on $10 \mathrm{Jul} 2017$

HAL is a multi-disciplinary open access archive for the deposit and dissemination of scientific research documents, whether they are published or not. The documents may come from teaching and research institutions in France or abroad, or from public or private research centers.
L'archive ouverte pluridisciplinaire HAL, est destinée au dépôt et à la diffusion de documents scientifiques de niveau recherche, publiés ou non, émanant des établissements d'enseignement et de recherche français ou étrangers, des laboratoires publics ou privés. 


\title{
COMMUNICATIONS
}

\section{The ground state tunneling splitting of malonaldehyde: Accurate full dimensional quantum dynamics calculations}

\author{
Maurício D. Coutinho-Neto \\ Theoretische Chemie, TU München, Lichtenbergstr. 4, D-85747 Garching, Germany
}

Alexandra Viel

Theoretische Chemie, TU München, Lichtenbergstr. 4, D-85747 Garching, Germany and LPQ-IRSAMC, Universite P. Sabatier, 118 route de Narbonne, F-31062 Toulouse, France

\author{
Uwe Manthe ${ }^{\mathrm{a})}$ \\ Theoretische Chemie, TU München, Lichtenbergstr. 4, D-85747 Garching, Germany \\ and Theoretische Chemie, Fakultät für Chemie, Universität Bielefeld, Postfach 100131, \\ D-33501 Bielefeld, Germany
}

(Received 8 September 2004; accepted 20 September 2004)

\begin{abstract}
Benchmark calculations of the tunneling splitting in malonaldehyde using the full dimensional potential proposed by Yagi et al. [J. Chem. Phys. 115, 10647 (2001)] are reported. Two exact quantum dynamics methods are used: the multiconfigurational time-dependent Hartree (MCTDH) approach and the diffusion Monte Carlo based projection operator imaginary time spectral evolution (POITSE) method. A ground state tunneling splitting of $25.7 \pm 0.3 \mathrm{~cm}^{-1}$ is calculated using POITSE. The MCTDH computation yields $25 \mathrm{~cm}^{-1}$ converged to about $10 \%$ accuracy. These rigorous results are used to evaluate the accuracy of approximate dynamical approaches, e.g., the instanton theory. (C) 2004 American Institute of Physics. [DOI: 10.1063/1.1814356]
\end{abstract}

\section{INTRODUCTION}

Due to its strong intramolecular hydrogen bond and amenable size malonaldehyde (MA) has been a very popular benchmark system for the study of intramolecular proton transfer. Early experimental investigations on the tunneling splitting $^{1-4}$ on MA sparked a series of theoretical studies aiming to understand the nature of its ground vibrational state. The early theoretical works of Carrington and Miller ${ }^{5,6}$ have shown that a one-dimensional effective treatment of the proton tunneling on MA is fundamentally flawed. Since then the determination of the ground state tunneling splitting of MA has been the focus of several studies ranging from reduced dimensionality quantum calculations $\mathrm{s}^{7,8}$ to elaborate full-dimensional semiclassical treatments. ${ }^{9-17}$ Most of these studies employed MA as a test case for method development measuring the accuracy of the calculation by comparing the computed value of the ground state splitting with the experimental one.

While most work on MA relied on semiempirical potential energy surfaces (PESs), recently Yagi et al. ${ }^{15}$ succeeded in constructing a full 21-dimensional ab initio PES. This progress has been facilitated by the use of the modified Shepard interpolation scheme introduced by Collins and co-workers. ${ }^{18-24}$ However, due to the numerical effort for constructing a full-dimensional PES, the ab initio level has been restricted to the second-order Møller-Plesset perturbation theory. Thus the potential barrier height for hydrogen

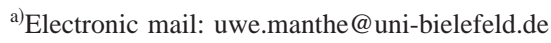

transfer of $3.2 \mathrm{kcal} / \mathrm{mol}$ is slightly low compared to higher level electronic structure methods $(3.8 \mathrm{kcal} / \mathrm{mol}$ for $\mathrm{CCSD}(\mathrm{T}) /\left(\right.$ aug-)cc-PVTZ ${ }^{17}$ and $4.3 \mathrm{kcal} / \mathrm{mol}$ for the composite $\mathrm{G} 2$ calculation from Barone and Adamo ${ }^{25}$ ).

Up to now, tunneling splittings based on this PES have been obtained within semiclassical approximations. Yagi et al. ${ }^{15}$ used the semiclassical approach of Makri and Miller $^{26}$ and found a tunneling splitting value of $13.9 \mathrm{~cm}^{-1}$. Using a more elaborate approach based on instanton theory, Mil'nikov et al. ${ }^{17}$ obtained a value of $30.7 \mathrm{~cm}^{-1}$. Moreover, since these instanton theory calculations can be performed on the fly (without requiring a predefined global PES), Mil'nikov et al. ${ }^{16,17}$ calculated the tunneling splitting of MA based on a higher $a b$ initio level. In these calculations they obtained values of 21.2 and $22.2 \mathrm{~cm}^{-1}$ depending on the computational details. These tunneling splittings are in remarkably good agreement with the experimental value of $21.6 \mathrm{~cm}^{-1}$.

When using approximate dynamical methods, judging the accuracy of the dynamics calculation only by comparison with experiment carries the risk of fortunate compensation of errors resulting from the dynamics and from the $a b$ initio potential. Since, to our knowledge, no accurate fulldimensional quantum mechanical calculation has been performed for the ground state splitting of MA to date, the present work attempts to provide such benchmark data. To this end, we employ two distinct quantum methodologies for the computation of the ground state tunneling splitting $\left(\Delta_{0}\right)$ of MA: namely, the POITSE and the MCTDH methodologies. The POITSE ${ }^{27}$ method is based on the stochastic diffu- 
sion Monte Carlo algorithm ${ }^{28-31}$ and allows the determination of excited states of high-dimensionality system. ${ }^{32}$ The $\mathrm{MCTDH}^{33,34}$ method has successfully been applied to study wave-packet dynamics in systems with a large number of degrees of freedom (see for example Refs. 35-38).

Both exact quantum dynamics methods require a predefined global PES because on the fly calculations are computationally prohibitive. Thus the present study employs the PES of Yagi et al. ${ }^{15}$ Due to the limited accuracy of this PES, we can not expect to find perfect agreement between the computed and experimental values. However, since the PES should be qualitatively or even semiquantitatively correct, the comparison of accurate and approximate dynamical results should be representative. Confirming the accuracy of the instanton approach for this particular PES may validate the applicability of this method also for a more quantitative (on the fly) PESs.

\section{SYSTEM}

MA is described using the 21 normal modes, $\mathbf{Q}$ $=\left\{Q_{1}, \ldots, Q_{21}\right\}$, calculated at the transition state of the proton transfer reaction ( $C_{2 v}$ symmetry). $Q_{21}$ corresponds to the hydrogen transfer mode and shows an imaginary frequency in the normal mode analysis. The choice of transition state normal modes allows for an equivalent description of both local minima of the potential energy surface and an efficient use of symmetry in the calculations. Considering mixed derivatives in the kinetic energy operator resulting from vibrational angular momenta irrelevant, the Hamiltonian for vanishing total angular momentum $(J=0)$ reads

$$
\hat{H}=-\frac{\hbar^{2}}{2} \sum_{i=1}^{21} \frac{\partial^{2}}{\partial Q_{i}^{2}}+V(\mathbf{Q}),
$$

where $V(\mathbf{Q})$ is the PES of Yagi et al. ${ }^{15}$ This form of the Hamiltonian is employed in the POITSE calculations. To enhance the convergence of the MCTDH calculations, there a transformed set of coordinates $\widetilde{\mathbf{Q}}$ is used:

$$
\begin{aligned}
& \widetilde{Q}_{i}=Q_{i}-F_{i}\left(Q_{21}\right), \quad i=1, \ldots, 20, \\
& \widetilde{Q}_{21}=Q_{21},
\end{aligned}
$$

where the $F_{i}$ are functions of the hydrogen transfer mode $Q_{21}$. The functions $F_{i}$ are chosen to result in $\widetilde{Q}_{i}, i$ $=1, \ldots, 20$ coordinate values which approximately vanish along the reaction path. The resulting new coordinates $\widetilde{\mathbf{Q}}$ decrease the correlation between the hydrogen transfer mode and the remaining coordinates. The transformed kinetic energy operator reads

$$
\begin{aligned}
\hat{T}= & -\frac{\hbar^{2}}{2}\left\{\sum_{i=1}^{21} \frac{\partial^{2}}{\partial \widetilde{Q}_{i}^{2}}+\sum_{i, j=1}^{20} \frac{\partial F_{i}}{\partial Q_{21}} \frac{\partial F_{j}}{\partial Q_{21}} \frac{\partial^{2}}{\partial \widetilde{Q}_{i} \partial \widetilde{Q}_{j}}\right. \\
& \left.-2 \sum_{j=1}^{20}\left(\frac{\partial F_{j}}{\partial Q_{21}} \frac{\partial}{\partial \widetilde{Q}_{21}}+\frac{\partial^{2} F_{j}}{\partial Q_{21}^{2}}\right)\right\} .
\end{aligned}
$$

\section{POITSE}

Due to their polynomial scaling with respect to dimensionality, stochastic methods like diffusion Monte Carlo $(\mathrm{DMC})^{28-31}$ are methods of choice for the quantum determination of bosonic ground state of large systems. The computation of excitation energies is however more complex but can be done using the POITSE ${ }^{27}$ methodology. DMC and POITSE algorithms have been extensively described in literature and will only be briefly reviewed focusing instead on the specific details for their application to MA.

The working equation in DMC with importance sampling for the 21-dimensional MA system is given by

$$
\begin{aligned}
\frac{\partial f(\mathbf{Q})}{\partial \tau}= & \sum_{j=1}^{21} \frac{\hbar^{2}}{2}\left\{\frac{\partial^{2}}{\partial Q_{j}^{2}} f(\mathbf{Q})-\frac{\partial}{\partial Q_{j}}\left[f(\mathbf{Q}) F_{j}(\mathbf{Q})\right]\right\} \\
& -\left[E_{l}(\mathbf{Q})-E_{r e f}\right] f(\mathbf{Q}),
\end{aligned}
$$

where the product function $f(\mathbf{Q})=\Psi(\mathbf{Q}) \Psi_{T}(\mathbf{Q})$ contains a guiding function $\Psi_{T}(\mathbf{Q})$ which approximates the solution $\Psi(\mathbf{Q})$ of the Schrödinger equation. In the above equation, the local energy $E_{l}(\mathbf{Q})=\Psi_{T}(\mathbf{Q})^{-1} \hat{H} \Psi_{T}(\mathbf{Q})$ and the quantum force $F_{j}(\mathbf{Q})=\nabla_{j} \ln \left|\Psi_{T}(\mathbf{Q})\right|^{2}$ notations have been used. A random walk technique is used to determine the steady state of Eq. (4). An ensemble of walkers is propagated from some arbitrary initial distribution using the short time approximation of the Green's function appropriate to Eq. (4).

In the POITSE scheme, excited state energies are extracted from the two-sided inverse Laplace transform of an imaginary time correlation function $\widetilde{\kappa}(\tau)$. The time dependent decay of this correlation function is computed using a multidimensional Monte Carlo integration combined with zero temperature DMC sidewalks. ${ }^{27}$ The decay of the correlation function $\widetilde{\kappa}(\tau)$ contains information about energy differences $E_{f}-E_{0}$ between ground $\left(E_{0}\right)$ and excited $\left(E_{f}\right)$ state. The renormalized POITSE correlation function can be written ${ }^{27}$ in a convenient form for Monte Carlo evaluation

$$
\widetilde{\kappa}(\tau)=\frac{\frac{\left\langle\Psi_{T}\left|A \exp \left[-\left(\hat{H}-E_{0}\right) \tau\right] A^{\dagger}\right| \Psi_{T}\right\rangle}{\left\langle\Psi_{T} \mid \Psi_{T}\right\rangle}}{\frac{\left\langle\Psi_{T}\left|\exp \left[-\left(\hat{H}-E_{0}\right) \tau\right]\right| \Psi_{T}\right\rangle}{\left\langle\Psi_{T} \mid \Psi_{T}\right\rangle}} .
$$

In this equation $A$ is a local operator chosen to project from the trial function $\left|\Psi_{T}\right\rangle$ onto some excited state $\left|\Psi_{f}\right\rangle$. An inverse Laplace transform of $\widetilde{\kappa}(\tau)$ yields the desired spectral information $\kappa(\omega)$. Since the tunneling splitting is the energy difference between the (symmetric) ground state and the lowest antisymmetric state (with respect to $Q_{21}$ ), a suitable projector $A$ for the computation of the tunneling splitting is the hydrogen transfer mode $Q_{21} \cdot{ }^{27}$

As trial function, a simple product of one-dimensional functions of the 21 normal modes

$$
\Psi_{T}^{(21 d)}(\mathbf{Q})=\prod_{i=1}^{21} \Phi_{i}\left(Q_{i}\right)
$$




$$
\begin{aligned}
& \Phi_{1}\left(Q_{21}\right)=\left[e^{-b_{21}\left(Q_{21}-a_{21}\right)^{2}}+e^{-b_{21}\left(Q_{21}+a_{21}\right)^{2}}\right] e^{-c_{21} Q_{21}^{4},} \\
& \Phi_{i}\left(Q_{i}\right)=e^{-b_{i}\left(Q_{i}-a_{i}\right)^{2}}, \quad i=1, \ldots, 20,
\end{aligned}
$$

has been used. The parameters of Eqs. (7) have been determined ${ }^{39}$ by a fit to the projections of the wave function along the 21 coordinates obtained via an unbiased DMC calculation ( $\left.\Psi_{T}(\mathbf{Q})=1\right)$. The evaluation of Eq. (5) is done by first creating an initial ensemble of walkers distributed as $\left(\Psi_{T}^{(21 d)}(\mathbf{Q})\right)^{2}$ using a variational Monte Carlo walk. Subsequently, DMC side walks are performed during which $\widetilde{\kappa}(\tau)$ is sampled. For each imaginary time step tested (from 20 down to $1 \mathrm{au}$ ) approximately 6500 decays have been created and then inverted using the maximum entropy method (see details in Ref. 40). An ensemble of 2000 walkers is used to create decays up to $\tau_{\text {final }} \simeq 15000$ a.u. In order to be able to compute long correlation decays needed for the extraction of small energy differences, two implementations of the DMC walk have been used: pure branching or combination of weights and branching. ${ }^{32,41}$ Both lead to the same value for the tunneling splitting. At the limit of small time step, the value of the tunneling splitting is found to be 25.7 $\pm 0.3 \mathrm{~cm}^{-1}$. The error bars have been estimated from the statistical noise of the decays as explained in Ref. 40. Additionally, the one reported here, includes the two results coming from the two different implementations of the DMC walk in POITSE employed here.

\section{MCTDH}

The tunneling splitting is computed by iteratively diagonalizing the Boltzmann operator $e^{-\beta \hat{H}}$. The multiconfigurational time-dependent Hartree (MCTDH) approach ${ }^{33,34}$ is employed to evaluate the action of the Boltzmann operator by the imaginary time propagation. For the iterative diagonalization, a Lanczos type scheme adapted for the MCTDH methodology is used. ${ }^{42}$ In order to utilize the inversion symmetry of the double well potential of malonaldehyde, the original scheme has been modified: the approach of Ref. 42 is used to create a Krylov-type basis localized dominantly in only one well. Then the inversion operator is applied to these basis states to generate an equivalent basis set localized in the other well and the $e^{-\beta \hat{H}}$ operator is diagonalized in the combined basis. This modified iterative diagonalization scheme allows to converge the calculation with less iterations and a smaller number of single-particle functions. A more extended description of the scheme will be given later in a full paper.

In the present computations, the correlation (CDVR) scheme $^{43}$ has been used for the evaluation of the potential energy matrix elements in the MCTDH representation and a scheme utilizing the ideas of Beck and Meyer ${ }^{44}$ has been employed for integrating the MCTDH equations of motion in time. In the Boltzmann operator employed in the iterative diagonalization, a $\beta$ value of 1000 a.u. has been used. The construction of local wavefunctions required in the modified iterative diagonalization approach has been achieved by choosing an initial wavefunction localized in one side of the double well potential and multiplying the wavefunction by a
TABLE I. Representation of the MCTDH wavefunction. $n$ is the number of single particle function, and $N$ is the size of the underlying time-independent basis set.

\begin{tabular}{cccccc}
\hline \hline coord. & $n$ & $N$ & coord. & $n$ & $N$ \\
\hline$\widetilde{Q}_{1}$ & 1 & 12 & $\widetilde{Q}_{11}$ & 1 & 12 \\
$\widetilde{Q}_{2}$ & 1 & 12 & $\widetilde{Q}_{12}$ & 1 & 12 \\
$\widetilde{Q}_{3}$ & 1 & 12 & $\widetilde{Q}_{13}$ & 2 & 13 \\
$\widetilde{Q}_{4}$ & 3 & 16 & $\widetilde{Q}_{14}$ & 2 & 13 \\
$\widetilde{Q}_{5}$ & 3 & 16 & $\widetilde{Q}_{15}$ & 1 & 18 \\
$\widetilde{Q}_{6}$ & 1 & 12 & $\widetilde{Q}_{16}$ & 2 & 13 \\
$\widetilde{Q}_{7}$ & 1 & 12 & $\widetilde{Q}_{17}$ & 3 & 24 \\
$\widetilde{Q}_{8}$ & 1 & 12 & $\widetilde{Q}_{18}$ & 1 & 16 \\
$\widetilde{Q}_{9}$ & 1 & 12 & $\widetilde{Q}_{19}$ & 3 & 27 \\
$\widetilde{Q}_{10}$ & 4 & 21 & $\widetilde{Q}_{20}$ & 3 & 32 \\
& & & $\widetilde{Q}_{21}$ & 6 & 48 \\
\hline \hline
\end{tabular}

Heaviside function in the $\mathrm{H}$ transfer coordinate $\widetilde{Q}_{21}$ before each iteration step. For the representation of the singleparticle functions, FFT schemes have been employed in coordinates $\widetilde{Q}_{20}$ and $\widetilde{Q}_{21}$ (grid ranges $[-100,100]$ and [-95,95], respectively) while Hermite DVRs have been used in all other coordinates. Grid sizes are given in Table I.

Extensive convergence tests have been carried out to determine the number of single-particle functions $n$ required in the MCTDH representation. Due to the extent of the material, a complete description of these tests can not be given in this communication. Details will be presented in a subsequent article. The most accurate MCTDH calculation employed the single-particle function basis given in Table I and obtained a tunneling splitting of $25 \mathrm{~cm}^{-1}$. Based on the convergence tests performed, this number is estimated to be converged to about $10 \%$ accuracy, i.e., within an uncertainty of 2 to $3 \mathrm{~cm}^{-1}$.

\section{DISCUSSION}

The first exact full quantum determinations in full dimensionality (21D) of the ground state tunneling splitting of malonaldehyde have been reported here. Two completely independent exact quantum methods, POITSE and MCTDH, have been used. Employing MCTDH, a tunneling splitting of $25 \mathrm{~cm}^{-1}$ has been computed. This number is estimated to be converged within approximately 2 to $3 \mathrm{~cm}^{-1}$ accuracy. The POITSE method results in an even more precise value of $25.7 \pm 0.3 \mathrm{~cm}^{-1}$. Thus, the POITSE method seems to be superior for obtaining ground state tunneling splittings. However, MCTDH offers also the possibility to study excited vibrational states which are more difficult to describe within the POITSE scheme. Such results will be presented in future work.

Comparing the theoretical tunneling splitting obtained for the Yagi et al. ${ }^{15}$ PES with the experimental value of $21.6 \mathrm{~cm}^{-1}, 45$ the theoretical splitting is found to be about $20 \%$ too high. Given that the potential barrier of this PES is known to be too small, this difference is hardly surprising. Still given an error in the barrier height of about $0.6 \mathrm{kcal} /$ mol, this difference seems rather small. 
The present calculation provides an important benchmark to be used for comparison with results from approximate dynamical methods. Such approximate methods might have a wider range of applicability by, e.g., allowing the use of on the fly techniques. As an example of such a comparison, the accurate tunneling splitting of $25.7 \pm 0.3 \mathrm{~cm}^{-1}$ obtained in the present work can be compared to the value of $30.7 \mathrm{~cm}^{-1}$ computed by Mil'nikov et al. ${ }^{17}$ using the instanton approach. The instanton approach overestimates the tunneling splitting by about $20 \%$. Given the simplicity of the instanton approach compared to rigorous quantum dynamics calculations, this should be considered as a rather good agreement. Thus, for the first time, the present work enables an unbiased evaluation of the accuracy of the instanton approach for a complex and realistic PES.

\section{ACKNOWLEDGMENTS}

Financial support by the Deutsche Forschungsgemeinschaft and the Fond der Chemischen Industrie are gratefully acknowledged. A.V. acknowledges financial support via a Marie Curie fellowship of the European Community program Improving Human Research Potential and the Socioeconomic Knowledge Base under the Contract No. HPMFCT-2000-00840. The Leibniz-Rechenzentrum, HLRB, and IDRIS (Project No. 41700 CP8) are acknowledged for the large amount of computing power provided. The authors would also like to thank K. Yagi, T. Taketsugu, and K. Hirao for kindly providing the source code for the MA potential.

${ }^{1}$ S. L. Baughcum, R. W. Duerst, W. F. Rowe, Z. Smith, and E. B. Wilson, J. Am. Chem. Soc. 103, 6296 (1981).

${ }^{2}$ P. Turner, S. L. Baughcum, S. L. Coy, and Z. Smith, J. Am. Chem. Soc. 106, 2265 (1984).

${ }^{3}$ S. L. Baughcum, Z. Smith, E. B. Wilson, and R. W. Duerst, J. Am. Chem. Soc. 106, 2260 (1984).

${ }^{4}$ D. W. Firth, K. Beyer, M. A. Dvorak, S. W. Reeve, A. Grushow, and K. R. Leopold, J. Chem. Phys. 94, 1812 (1991).

${ }^{5}$ T. Carrington and W. H. Miller, J. Chem. Phys. 81, 3942 (1984).

${ }^{6}$ T. Carrington and W. H. Miller, J. Chem. Phys. 84, 4364 (1986).

${ }^{7}$ N. Shida, P. F. Barbara, and J. E. Almlöf, J. Chem. Phys. 91, 4061 (1989).

${ }^{8}$ D. Babić, S. D. Bosanac, and N. Došlić, Chem. Phys. Lett. 358, 337 (2002).

${ }^{9}$ T. D. Sewell and D. L. Thompson, Chem. Phys. Lett. 193, 347 (1992).

${ }^{10}$ Y. Guo, T. D. Sewell, and D. L. Thompson, Chem. Phys. Lett. 224, 470 (1994).

${ }^{11}$ M. Ben-Nun and T. J. Martínez, J. Phys. Chem. A 103, 6055 (1999).

${ }^{12}$ Z. Smedarchina, W. Siebrand, and M. Z. Zgierski, J. Chem. Phys. 103, 5326 (1995).
${ }^{13}$ T. D. Sewell, Y. Guo, and D. L. Thompson, J. Chem. Phys. 103, 8557 (1995).

${ }^{14}$ V. A. Benderskii, E. V. Vetoshkin, I. S. Irgibaeva, and H. P. Trommsdorff, Chem. Phys. 262, 393 (2000).

${ }^{15}$ K. Yagi, T. Taketsugu, and K. Hirao, J. Chem. Phys. 115, 10647 (2001).

${ }^{16}$ G. V. Mil'nikov, K. Yagi, T. Taketsugu, H. Nakamura, and K. Hirao, J. Chem. Phys. 119, 10 (2003).

${ }^{17}$ G. V. Mil'nikov, K. Yagi, T. Taketsugu, H. Nakamura, and K. Hirao, J. Chem. Phys. 120, 5036 (2004)

${ }^{18}$ J. Ischtwan and M. A. Collins, J. Chem. Phys. 100, 8080 (1994).

${ }^{19}$ M. J. T. Jordan, K. C. Thompson, and M. A. Collins, J. Chem. Phys. 102, 5647 (1995).

${ }^{20}$ M. J. T. Jordan, K. C. Thompson, and M. A. Collins, J. Chem. Phys. 103, 9669 (1995)

${ }^{21}$ M. J. T. Jordan and M. A. Collins, J. Chem. Phys. 104, 4600 (1996).

${ }^{22}$ K. C. Thompson and M. A. Collins, J. Chem. Soc., Faraday Trans. 93, 871 (1997).

${ }^{23}$ K. C. Thompson, M. J. T. Jordan, and M. A. Collins, J. Chem. Phys. 108, 564 (1998).

${ }^{24}$ R. P. A. Bettens and M. A. Collins, J. Chem. Phys. 111, 816 (1999).

${ }^{25}$ V. Barone and C. Adamo, J. Chem. Phys. 105, 11007 (1996).

${ }^{26}$ N. Makri and W. H. Miller, J. Chem. Phys. 91, 4026 (1989).

${ }^{27}$ D. Blume, M. Lewerenz, P. Niyaz, and K. B. Whaley, Phys. Rev. E 55, 3664 (1997).

${ }^{28}$ M. A. Suhm and R. O. Watts, Phys. Rep. 204, 293 (1991).

${ }^{29}$ C. J. Umrigar, M. P. Nightingale, and K. J. Runge, J. Chem. Phys. 99, 2865 (1993)

${ }^{30}$ B. L. Hammond, W. A. Lester, and P. J. Reynolds, Monte Carlo Methods in ab initio Quantum Chemistry (World Scientific, Singapore, 1994).

${ }^{31}$ K. B. Whaley, Advances in Molecular Vibrations and Collision Dynamics, Vol. III, edited by J. Bowman and Z. Bačić (Academic, JAI Press, Inc., 1998).

${ }^{32}$ A. Viel and K. B. Whaley, J. Chem. Phys. 115, 10186 (2001).

${ }^{33}$ H.-D. Meyer, U. Manthe, and L. S. Cederbaum, Chem. Phys. Lett. 165, 73 (1990).

${ }^{34}$ U. Manthe, H. D. Meyer, and L. S. Cederbaum, J. Chem. Phys. 97, 3199 (1992).

${ }^{35}$ M. H. Beck, A. Jackle, G. A. Worth, and H. D. Meyer, Phys. Rep., Phys. Lett. 324C, 1 (2000).

${ }^{36}$ F. Huarte-Larranaga and U. Manthe, J. Chem. Phys. 113, 5115 (2000).

${ }^{37}$ H. B. Wang and M. Thoss, J. Chem. Phys. 119, 1289 (2003).

${ }^{38}$ T. Wu and U. Manthe, J. Chem. Phys. 119, 14 (2003).

${ }^{39}$ The parameter values are available upon request to the authors.

${ }^{40}$ P. Huang and K. B. Whaley, Phys. Rev. B 67, 155419 (2003).

${ }^{41}$ P. Huang, A. Viel, and K. B. Whaley, in Recent Advances in Quantum Monte Carlo Methods, Part II, edited by W. A. Lester, Jr., S. M. Rothstein, and S. Tanaka (World Scientific, Singapore, 2002), Vol. 2 of Recent Advances in Computational Chemistry, p. 111.

${ }^{42}$ U. Manthe and F. Matzkies, Chem. Phys. Lett. 252, 71 (1996).

${ }^{43}$ U. Manthe, J. Chem. Phys. 105, 6989 (1996).

${ }^{44}$ M. H. Beck and H. D. Meyer, Z. Phys. D: At., Mol. Clusters 42, 113 (1997).

${ }^{45}$ T. Baha, T. Tanaka, I. Morino, K. M. Yamada, and K. Tanaka, J. Chem. Phys. 110, 4131 (1999). 